\title{
¿HACIA DÓNDE VA LA HISTORIA DE LA ENFERMERÍA?
}

\author{
Carmen González Canalejo \\ Josefa MÁrquez MEMBrive \\ Universidad de Almería
}

\section{RESUMEN}

El propósito de este artículo, en términos generales, consiste en hacer un balance de las líneas de estudio en la Historia de la Enfermería valorando los posibles temas de interés que puedan ser investigables y, de otro lado, analizar qué aportaciones pueden ofrecer y de hecho están ofreciendo, los estudios de este campo a la historiografía general

Palabras clave: historia de la enfermeria

\section{TOWARDS WHERE THE NURSING HISTORY IS GOING?}

\section{SUMMARY}

The objectives of this article are, in general terms, to do a balance of the lines of study in the Nursing History valuing the possible subjects of interest that can be investigables and, also we try to analyze what contributions can offer and in fact they are offering, the studies from this field to the general historiography.

Keywords: Nursing history

Los objetivos de esta comunicación son, en términos generales, hacer un balance de las líneas de estudio en la Historia de la Enfermería valorando los posibles temas de interés que puedan ser investigables y, de otro lado, analizar qué aportaciones pueden ofrecer y de hecho están ofrecien- do, los estudios de este campo a la historiografía general, de acuerdo con las corrientes historiográficas del momento.

Como material y métodos se ha llevado a cabo un análisis descriptivo de los trabajos presentados en los congresos y jornadas de Historia de la Enfermería Española celebradas en los últimos diez años. En segundo lugar, se ha utilizado material bibliográfico de interés como fuente de estudio, bien por ser trabajos derivados de las escasas tesis doctorales que están relacionadas con aspectos de los cuidados, bien por servir de soporte metodológico para el uso de las nuevas corrientes en la Historia.

El desarrollo análitico de hacia dónde va la Historia de la Enfermería española lo vamos a tratar bajo tres aspectos:

Primer aspecto: evolución de los temas tratados en las dos últimas décadas:

Para situarnos, haremos un breve balance sobre algunos trabajos publicados a raíz de los ochenta $y$, que a nuestro juicio, han sido significativos; a menudo, por ser las únicas referencias de las que muchos docentes enfermeros/as hemos podido disponer, al menos en los primeros años $y$, que a pesar de las deficiencias, con frecuencia metodológicas que hoy en día se les puede achacar, sin duda alguna, representaron como mínimo un estímulo para que algunos hayamos optado por el estudio del conocimiento histórico en el campo de la salud y específicamente de los cuidados enfermeros. Más adelante, intentaré abordar la conexión 
de la Historia de la Enfermeria con la Historia Global'.

\section{El comienzo de los 80}

En nuestro país, el interés por los temas de historia, igual que ocurre con los aspectos metodológicos de la enfermería, arranca a partir de los años 80 , tiene un ascenso en los $90 \mathrm{y}$, esperemos que se consolide en esta presente década.

El primer avance parte de los planes de estudios de enfermería que desde principios de los 90 , marca la tendencia de separar la materia de Historia de la Enfermería que hasta estos momentos estaba englobada en la Enfermería Básica y se configura, como muestra del interés científico para el avance en el campo de los cuidados, en una asignatura propia con denominación y descriptores específicos.

Hasta esta circunstancia, la anemia de los trabajos publicados era más que evidente ya que, aparte de la manoseada versión en castellano del tratado de Patricia Donahué, fue el trabajo producto de la tesis de Domínguez Alcón uno de los de mayor interés que, trata de seguir la evolución de los cuidados en España desde una perspectiva, centrada en los hospitales medievales, donde se analizan diferentes aspectos, ligados al trabajo de los cuidadores y a la atención de los enfermos dentro de los hospitales. El análisis de este estudio es sin duda valioso, pero hoy día, escaso en cuanto a la Historia Contemporánea de la Enfermería ya que está demasiado centrado en los aspectos sociológicos de los cuidadores y de las instituciones. Sin duda, es un estudio significativo para el contexto de los trabajos realizados en los 80; pero hoy día, necesitariamos ampliar este marco y articularlo con una panorámica historiográfica global que nos ayude a hilvanar los aspectos políticos, económicos y de conflicto social que han coexistido con el funcionamiento de los establecimientos sanitarios, administradores de los cuidados, servicios que prestaban los cuidadores y su relación con las estructuras de poder.

Una publicación que apareció traducida al castellano en los 80, fue un trabajo de Ehrenrich y English ${ }^{+}$. Son una serie de textos agrupados en forma de cuaderno y que, por vez primera, aportaron una perspectiva antropológica a la Historia de la Enfermería. Cumple el importante papel que debe cumplir la Historia que es la función de explicar para ayudarnos a los cuidadores a comprender nuestro papel y poder producir cambios democratizadores en la estructura sanitaria. En este sentido, los textos ponen en evidencia las distintas formas de exclusión y manipulación vividas por las mujeres en contacto con la institución médica. Las profundas reflexiones acerca del papel de subordinación y absolutamente secundario de matronas y enfermeras, frente a la hegemonía médica en los últimos siglos, como resultado de la discriminación de las trabajadoras de la sanidad por razones de sexo, nos abren una puerta para tratar el tema de la ideología de género en el campo de la salud desde la corriente de la Historia Antropológica que es la perspectiva que hoy cabría complementar.

Esta década, finalizó con la constitución en el año 1989 del Seminario Permanente de Historia de la Enfermería, promovido por la EUE de la Universidad Complutense de Madrid y con la participación española en el Proyecto del Consejo

\footnotetext{
El concepto de "historia global" queda claramente definido por Josep Fontana en La Historia, ¿para qué?, ponencia presentada en Granada el día 27-X-1996, donde habla de la necesidad de un modelo globalizador que, a menudo, la Historia postmoderna, reemplaza por las excesivas "historias sectoriales" como consecuencia de la diversificación y alejamiento de una hisıoria total.

P. Donahue, Historia de la Enfermeria, Barcelona, 1985, versión española.

C. Domínguez Alcón, Los cuidados y la profesión enfermera en España, Madrid, Pirámide, 1986. La autora relleja en este trabajo una relación descriptiva bastante amplia sobre las instituciones hospitalarias en España a partir del siglo X.

" B. Ehrenreich y D. English, Brujas, comadronas y enfermeras. Historia de las sanadoras. Dolencias y trastomos. Politica sexual de la enfermedad, Barcelona, laSal, edicions de les dones, 1988
} 
Internacional de Enfermería (CIE) para el año 1999: La Historia de la Enfermería en los últimos dos siglos.

\section{El avance en los años 90}

El interés científico por la Historia de la Enfermería en estos años, no sólo es evidente por el aumento en el número de trabajos publicados y aparición de revistas específicas, sino también por la celebración de congresos, jornadas y seminarios relativos a la historia de los cuidados.

Una de las publicaciones bibliográficas de los 90, es el trabajo publicado por Eseverri Chaverri que muestra curiosidad por bucear en la Historia de Enfermería hispanoamericana, aunque falta profundización en este aspecto y, sobre todo, historia comparativa. Numerosos textos que muestra el autor son significativos porque nos dejan ver los entresijos de la vida interna de los hospitales y las distintas órdenes que se dedicaban al cuidado de los enfermos y marginados, asi como de los estatutos y normas que regulaban su funcionamiento interno".

Otro estudio de mediados de los 90 es el capítulo de M. Santo Tomás que proporciona una reflexión global acerca de las causas que han favorecido el hecho de que la profesionalización de los enfermeros, dentro de un marco laboral y de laicización de las enseñanzas a los cuidadores, se haya realizado mucho más tarde que en el resto de los países de la cultura occidental ${ }^{5}$.
Por otra parte, el control que la Iglesia ha ejercido, a lo largo de los siglos, tanto en las enseñanzas enfermeras como en la administración de las instituciones prestadoras de cuidados, ha quedado patente en algunos análisis que se han realizado en la Historia de la Enfermería”. Pero cabría indicar que, a menudo falta objetividad respecto al tema eclesiástico del que, a mi modo de ver, está casi todo por hacer. Un ejemplo de ello es la escasez de estudios comparativos respecto al modelo pedagógico de las escuelas no católicas europeas en relación a las escuelas de los países de la cuenca mediterránea, las cuales siguieron bajo el control de la Iglesia católica de forma ininterrumpida a lo largo de la historia; de esta forma, podríamos entrar de lleno en las causas que frenaron el proceso de profesionalización e institucionalización de las enseñanzas en nuestro país ${ }^{8}$.

Logrados unos primeros horizontes, llamémosle de arranque, de los estudios de la Historia de la Enfermería, en octubre de 1991 y, tras la celebración del I Seminario de Historia de Enfermería, se contempló trabajar las distintas fases del Proyecto de Investigación de la Historia de la Enfermería en España donde se acordó que en cada año se cubriría un periodo $y$, al final, se expondrían los trabajos en jornadas, congresos o reuniones científicas?.

\section{'C. Eseverri Chaverri, Historia de la enfermeria española e hispanoamericana, Madrid, Univérsitas, 1995.}

'M. Santo Tomás en C. Fernández Ferrin y otros, Máster de enfermería. Enfermería Fundamental, Barcelona, Masson, 1994, pp. 3-131.

' Una muestra de análisis de las influencias de la Iglesia en el desarrollo de la profesionalización de la enfermería es el realizado por J. Hernández Conesa, Historia de la enfermeria. Un análisis histórico de los cuidados de enfermeria, Madrid, Interamericana McGraw-Hill, 1995.

* Un ejemplo de ello es el trabajo de F. Hernández Martín, (coord.), Historia de la enfemería en España que muestra una amplia descripción de la historia enfermera en nuestro pais (desde la antigüedad hasta nuestros días) pero sin el análisis objetivo que contrarreste la visión que la autora describe de las numerosas aportaciones de lglesia a la profesión enfermera, para la necesaria imparcialidad y rigor que cualquier estudio metodológico de la historia requiere.

- E. Calvo Charro y S. Garcia Barrios, (coord.), La enfermeria en España desde sus origenes hasta La Edad Media. Actas del II Congreso Nacional de Historia de la Enfermeria, Málaga, Universidad de Málaga, 1998, describen los objetivos del Seminario Permanente así como las fases del Proyecto sobre la Investigación de la Historia en España. Esta fuente nos permite ordenar los actos científicos llevados a cabo para la presentación de los trabajos correspondientes, así como el análisis de los distintos temas tratados en cada una de las fases de dicho proyecto. En las tres primeras fases se celebraron, además del I Seminario en Madrid, dos Jornadas Nacionales de Investigación celebradas en Madrid y Albacete en octubre de 1992 y 1993 respectivamente. 
Como resultado de las cuatro fases de este proyecto, se celebran, a lo largo de la década de los años 90 un total de ocho eventos científicos, incluyendo cuatro congresos nacionales de Historia de la Enfermería. La revisión de las actas y libros de resúmenes publicados, nos proporciona una panorámica de los temas tratados ${ }^{10}$ que se pueden agrupar de la siguiente forma" ${ }^{11}$

\section{Análisis de los distintos manuales publi-} cados, contenidos y cronología, que nos orientan sobre cómo han sido las enseñanzas de los cuidados.

2. Aspectos de la docencia relacionados con los contenidos de los planes de estudio, perfil docente, aparición de escuelas, funcionamiento, y corpus legislativo relacionado con los requisitos exigidos a los aspirantes para su acceso, así como estudios sobre normativas que regían el funcionamiento interno de las escuelas. Estos análisis descriptivos se han llevado a cabo en varias comunidades: Galicia, Castilla-La Mancha, Extremadura; también en la zona de Levante, Andalucía, Cataluña y Canarias; pero faltan estudios comparativos que nos permitan inferencias sobre aspectos comunes y generales relativos a la enseñanza que nos ayuden a descubrir los factores que dificultaron el reconocimiento científico y social de la actividad enfermera.

\section{Sobre los hospitales y la atención a los} enfermos es el grueso de los estudios realizados. Se recogen aspectos muy diversos tanto en localización (hospitales prácticamente de todas las comunidades españolas), así como en aspectos de normativas y reglamentos de funcionamiento, asistencia, perfil de los cuidadores, inventarios financieros y detalles del personal asalariado y congregaciones religiosas que administraban cuidados, inmuebles, estructura física de los mismos, perfil de pacientes que se atendían, cómo era la vida dentro de los hospitales etc.

4. Otros temas explorados en una proporción mucho menor, pero no por ello de menor interés, son los temas relacionados con el asociacionismo profesional como es el caso de algunos estudios locales de matronas y enfermeras, como los realizados en la Universidad de Alicante, también de practicantes, médicos, dentistas o farmacéuticos que, a veces, confluyen en intereses profesionales y entran en conflicto. Hay algunos estudios sobre este tema realizados a nivel local. Respecto a los temas relacionados con los aspectos políticos hay algunos estudios de enfermería durante la Guerra Civil, la Segunda República y el Sexenio.

La periodización de todos los trabajos presentados a lo largo de esta última década es muy amplia y abarca márgenes que van desde plena edad media hasta los hospitales de finales de la edad moderna y contemporánea; sin embargo, hay muy poco en historia antigua.

\section{Segundo aspecto: las corrientes historiográficas del momento y su aplicación a la Historia de la Enfermería}

Una idea clara que se desprende del análisis anterior, es que a pesar de los esfuerzos realizados en estos últimos años, en la Historia de los Cuidados está casi todo por hacer. Tenemos la suerte de tener una gran riqueza de documentos archivísticos aún sin explorar; ahora bien, aparte de los problemas metodológicos que debemos salvar en cualquier estudio, lo importante es emplear nuevos enfoques que cambien determinadas visiones que a veces, han constituído mitos en nuestra propia historia.

\footnotetext{
10 Ibídem.

"F. Hernández Martín, (coord.), Trabajo de investigación sobre la Historia de la Enfermeria en España en los siglos XVI y XVII. Actas del I Congreso Nacional de la historia de la enfermeria en España, Barcelona, Fundació Uriach, 1996. Véase también el Libro de resúmenes de las comunicaciones del III Congreso, celebrado en Mérida, 1997 y el libro de resúmenes del IV Congreso celebrado en Gijón, 1999.
} 
Una propuesta de reconstruir la historia enfermera es a través de la Historia Social. Si algún campo puede encajar en la Historia Social, ése es el campo de la salud y, muy especialmente los cuidados enfermeros; ante todo, porque la Historia de los Cuidados es una "historia desde abajo"12, cuyos actores y escenario, están en contacto directo con el mundo marginal de pobres, enfermos y desvalidos, con la gente de la calle que pierde la salud, a menudo, por la miseria y la falta de recursos. Es, además, la historia de ayudadores que simultanean las tareas de las instituciones hospitalarias en las que trabajan con la asistencia domiciliaria y, que interactúan con lo que hoy se denomina "el sujeto social"13.

Dentro de la Historia Social, las nuevas corrientes están relacionadas con la antropología que se interesa por los grupos, en especial, por las clases sociales que se conciben en términos de rivalidad, competencia o colaboración.

Natalie Zemón, pone de manifiesto las ventajas de esta nueva forma que, unida a la microhistoria (la microhistoria antropológica) ${ }^{14}$ nos va a permitir acercarnos a una historia local, una vivencia o experiencia familiar y también a los conflictos sociales. La relación entre la Antropología Social y la Historia Social es una conjunción que va a permitirnos un análisis más profundo y real en la Historia de la Enfermería al poder examinar al sujeto social desde unos elementos culturales que, a menudo, nos aclaran las relaciones de poder y dominación de unos grupos sobre otros ${ }^{15}$.

Una caracteristica de la Historia Social es que se puede aplicar a cualquier periodo. Aunque si bien es cierto que los trabajos hasta ahora publicados son, en su mayoría, de contemporánea, también se pueden realizar estudios de mundo antíguo, medieval y moderno.

Otra forma dentro de la Historia Social a la que hemos hecho alusión en la introduccion, es el asociacionismo contemporáneo ${ }^{16}$. Como elemento esencial, cabe destacar que, el derecho de asociación como derecho individual que aparece en el Sexenio de 1868 y queda recogido en la Constitución de 1869, permitió la creación de los colegios de practicantes y matronas que se asociaron como colectivos a partir de finales del XIX. Pero se necesitan mayores esfuerzos en investigación de materia asociativa que nos indique, hasta qué punto el asociacionismo de la Restauración, la República y las dictaduras de Primo de Rivera y franquista, está mediatizado por las oligarquías locales y/o el control de la Iglesia ${ }^{17}$.

${ }^{12}$ E. Hernández Sandoica, Los caminos de la Historia, Síntesis, Madrid, 1995, pp 79-89.

1" Un análisis de la evolución de la Historia Social en España es el que realiza C. Barros en "El retorno del sujeto social en la historiografía española". Véase S. Castillo y J. M. " Ortiz de Orduño (Coor), Estado, protestas y movimientos sociales, Bilbao, Asociación de Historia Social, Universidad del País Vasco, 1998, pp. 191-214.

${ }^{1+}$ N. Zemon, Las formas de la historia social, Historia Social, n. ${ }^{\circ}, 7$, primavera-verano 1990, pp. 177-182.

15 Véase, E. P. Thompson, Folklore, antropología e Historia Social, Historia Social, n. ${ }^{\circ} 1989$ pp. 97-103, donde el autor hace público una serie de objeciones en relación a la idea del estudio de la historia desde una perspectiva exclusivamente economicista, dejando a un lado los elementos culturales que son los que, en definitiva, van a poner de relieve la determinación de la conciencia social en la medida en que entran en juego las relaciones de dominio y subordinación en las que los hombres y mujeres se ven envueltos en el mecanismo de producción.

10 Pere Solá en Poderes y asociacionismo en la España contemporánea (siglos XIX y XX). Algunas consideraciones. El autor, realiza un análisis sobre la hipótesis de trabajo basada en la existencia de una relación entre la red de asociaciones y las estructuras de poder local y supra-local. Véase S. Castillo y J. M. a Ortiz de Orruño (coo), Estado, protesta y movimientos sociales, Bilbao, Asociación de Historia Social, Universidad del Pais Vasco, 1998, pp. 359-370.

${ }^{17}$ L.C. Navarro Pérez, conferencias impartidas en el curso de doctorado Metodologia para el estudio de las transformaciones sociales y politicas en el siglo XIX español. Universidad de Almeria, curso 1999/2000. En el análisis del material entregado, se debaten los temas y fuentes de interés en la acción colectiva según los trabajos presentados en los últimos congresos de Historia Social. 
Otra idea que se apunta como necesaria en las nuevas formas de hacer historia es explorar en la dimensión del género o historia de género ${ }^{18}$. Aquí cabe considerar que si bien los estudios de investigación de la mujer están avanzados en Europa en el año 1960; en España, hasta bien entrado 1980, no se empiezan a desarrollar; no obstante, en estos últimos cinco años se está empezando a profundizar en estudios que tratan de las condiciones específicas femeninas en el ámbito asociativo que perfilan cómo y en qué medida estas asociaciones profesionales impulsaron la educación, la higiene o la salud. Continúan faltando estudios que sitúen el contexto de las mujeres dentro del proceso de trabajo en el sentido de conciencia de clase trabajadora, que analicen los problemas de escalas salariales diferenciadas sexualmente y el enfoque de la vida de la familia trabajadora ${ }^{19}$.

\section{Tercer aspecto: aportación de la $\mathrm{H}^{\mathrm{a}}{ }^{\mathrm{a}}$ de los Cuidados a la historiografía general}

¿Cómo podemos aplicar estas corrientes a la investigación en la Historia de los Cuidados? Trataré muy brevemente dos perspectivas:

A) Propuesta de temas investigables en la Historia Enfermera:

El análisis de los trabajos aparecidos en las últimas décadas, así como la publicación de las primeras tesis doctorales, nos sirve para situarnos y abrir nuevas perspectivas para orientar, en los próximos años, nuestro campo de investigación; puesto que, el estudio de temas, desde nuestra perspectiva, debe ir acorde a las corrientes historiográficas comentadas. Los temas investigables que se proponen estarían dentro de las siguientes líneas:

- Desde la microhistoria antropológica se puede estudiar, la trilogía religión-enfermedad y remedios populares, o una experiencia determinada en el proceso de salud-enfermedad de un individuo, familia o grupo social; el estudio de la familia dentro de los procesos de trabajo; análisis específicos sobre la situación de las enfermeras dentro del mundo laboral, las jerarquías y capas dominantes en el mundo de la salud; el comportamiento de los pacientes ante la muerte o incluso, se pueden analizar los aspectos de salubridad de una comunidad teniendo la salud como indicador de bienestar social; por ejemplo, el estudio de los condicionamientos sociales y su relación con las epidemias y la higiene pública, en cualquier periodo de la historia. Otro aspecto de la Historia de Enfermería desde un abordaje antropológico sería el estudio de los mitos que se han generado respecto al rol de las mujeres cuidadoras como consecuencia de la ideología de género ${ }^{21}$.

- Temas de asociacionismo que, en un momento determinado pudieran actuar como punta de lanza de modernización social22. Habría que estu-

\footnotetext{
1s D. González Luna, profesora de la Universidad Autónoma de Barcelona presentó la ponencia Balance sobre los estudios de las mujeres: perspectivas de futuro, el día 27-4-2000 en la Universidad de Almería donde analizaba la necesidad de nuevos estudios de género que nos permitan valorar el concepto de género a través de la historia de la mujer, desde una perspectiva antropológica para mejorar las condiciones sociales (familia, cotidaneidad etc.), todo ello, planteado como fórmula de ver una historia global.

${ }^{19}$ Véase el ensayo de Joan W. Scott Sobre el lenguaje, el género y la historia de la clase obrera publicado en Historia Social, n. ${ }^{\circ} 4$ basado en el libro Gender and the Politics of History, New York, 1998 de la misma autora.

${ }^{20}$ M. D. Nogales, La sanidad municipal en la Valencia foral moderna (1479-1707), es una tesis doctoral leída en la Universidad de Valencia, curso 92/93 que recoge, a nivel local, tanto los aspectos sociales de la marginación como las medidas municipales encaminadas al fomento de la salud de los ciudadanos en la edad moderna.

${ }^{21}$ M-F. Collière, Promover la vida, Madrid, McGraw-Hil//Interamericana, 1993.

${ }_{22}^{2}$ J. Siles, Estructura familiar y función social de la mujer en Alicante (1868-1936), Alicante, Instituto de Cultura "Juan Gil-Albert", 1995 es resultado de una tesis doctoral sobre el tema de asociacionismo que puede servir de base para otros estudios locales contemporáneos comparativos acerca de colectivos sanitarios como es el caso de las matronas y practicantes de Alicante.
} 
diar hasta qué punto los colegios profesionales de practicantes, enfermeras y matronas sirvieron como motor impulsor de integración de las mujeres en el mundo laboral para analizar la diferencia de género y entender la historia de las enfermeras desde un modo algo distinto. También en la Historia de la Enfermería podemos analizar a intelectuales como Concepción Arenal o la misma Nightingale como precursoras de un movimiento a favor de una serie de medidas reformistas que afectaron a la educación de las mujeres de finales del siglo XIX. Por otro lado, el mundo de los cuidados nos puede mostrar que los colectivos sanitarios forman categorías bien definidas y darnos pistas acerca de la vida de la clase media, tan poco investigada y de la que poco se sabe en la historiografía española.

- Temas relacionados con el género como puede ser la evolución de los estudios exigidos para la obtención de los títulos de enfermeras/os y matronas como indicador de la ilustración general de las mujeres de clase media. Otro tema investigable, sería analizar el modelo pedagógico de las escuelas de enfermeras y matronas que se inicia en la Restauración ${ }^{23}$. Faltan estudios comparativos sobre los modelos pedagógicos de la corriente conservadora, tradicional y católica de las escuelas de enfermería y la corriente liberal de escuelas laicas que se inauguraron a finales del siglo XIX, de acuerdo con el movimiento de enseñanza para la mujer, destinado a "contribuir y fomentar las condiciones de vida para la mujer" y analizar en qué medida las enseñanzas de enfermería estuvieron mediatizadas por el control de la Iglesia y por las corrientes higienistas-positivistas del momento ${ }^{24}$.
B) Articular el papel del estado y las instituciones sanitarias

Algunas cuestiones que habría que indicar para la conexión con la historia global y que a menudo se echan en falta es, en primer lugar, la relación del estado con las instituciones hospitalarias y dispensarios en los que trabajan los sanitarios. La importancia de la acción colectiva viene dada por la conexión directa entre el nivel de quejas de un determinado colectivo y la aparición de movilizaciones, puesto que grupos con demandas moderadas y escasos recursos internos, pueden llegar a ponerse en movimiento y dicha acción colectiva no puede desligarse del papel del estado.

En este sentido, se echa en falta mayores referencias descriptivas acerca de los tipos de protesta sanitaria, conflictividad laboral de ámbito provincial, así como la descripción de la tipología de protesta.

En segundo lugar, analizar el funcionamiento de las instituciones sanitarias y de asistencia social en su relación con la oligarquía dominante. Los estatus jerárquicos que toman las decisiones de lo público, es otro aspecto necesario de investigar, y que está ausente, a pesar de la importancia que se le ha dado en enfermería a las viejas capas dominantes.

Otro aspecto relacionado con las instituciones y el estado, es ver en qué medida afectó el proceso de las desamortizaciones a los monasterios y hospitales controlados por la Iglesia así como analizar en qué medida las élites del poder sanitario se vieron implicadas en las redes de caciquismo que a nivel local se desarrolló desde la Restauración hasta $1931^{25}$

\footnotetext{
${ }^{23} \mathrm{~J}$. Carles Clemente La escuela universitaria de enfermeras de Madrid. Historia de una iniciativa humanitaria de la Cruz Roja Española (1918-1997), Madrid, Fundamentos colección ciencia, 1999.

${ }^{2+}$ M. Nash, Rojas. Las mujeres republicanas en la guerra civil, Madrid, Taurus, 1999, pp. 35-91.

${ }^{25}$ F. Martínez López, conferencias impartidas en el curso de doctorado Caciquismo, élites y centro de poder en la Restauración española, Universidad de Almería, curso 1999/2000, donde se analizan distintos aspectos relacionados con la sociedad española desde la Restauración hasta 1931 que nos sugieren temas investigables en el ámbito sanitario los cuales estén asociados a la corrupción, caciquismo y redes clientelares sociales de alcance local.
} 
A modo de conclusión, tan sólo señalar que estos tres aspectos que hemos analizado, son algunos ejemplos de temas investigados e investigables en la Historia de la Enfermería los cuales, desde nuestro punto de vista, pueden aportar una gran riqueza a la Historia general tanto por la variedad de los temas como por la cantidad de la documentación que aún está sin explorar.

La Historia de la Enfermería, si la estudiamos de forma cohesionada y global, podrá servir de herramienta de transformación social que proporcione un mayor impulso en el proceso democratizador en el área de la salud.

Una cuestión importante que Julio Aróstegui ${ }^{26}$ apunta como necesaria en la investigación de la Historia, es la realización de estudios de ámbito provincial o local para, de esta forma, unir a través de estudios comparativos, los nexos que nos permitan tener una visión integral de la Historia y nos ayude a ver mejor, la realidad del presente.

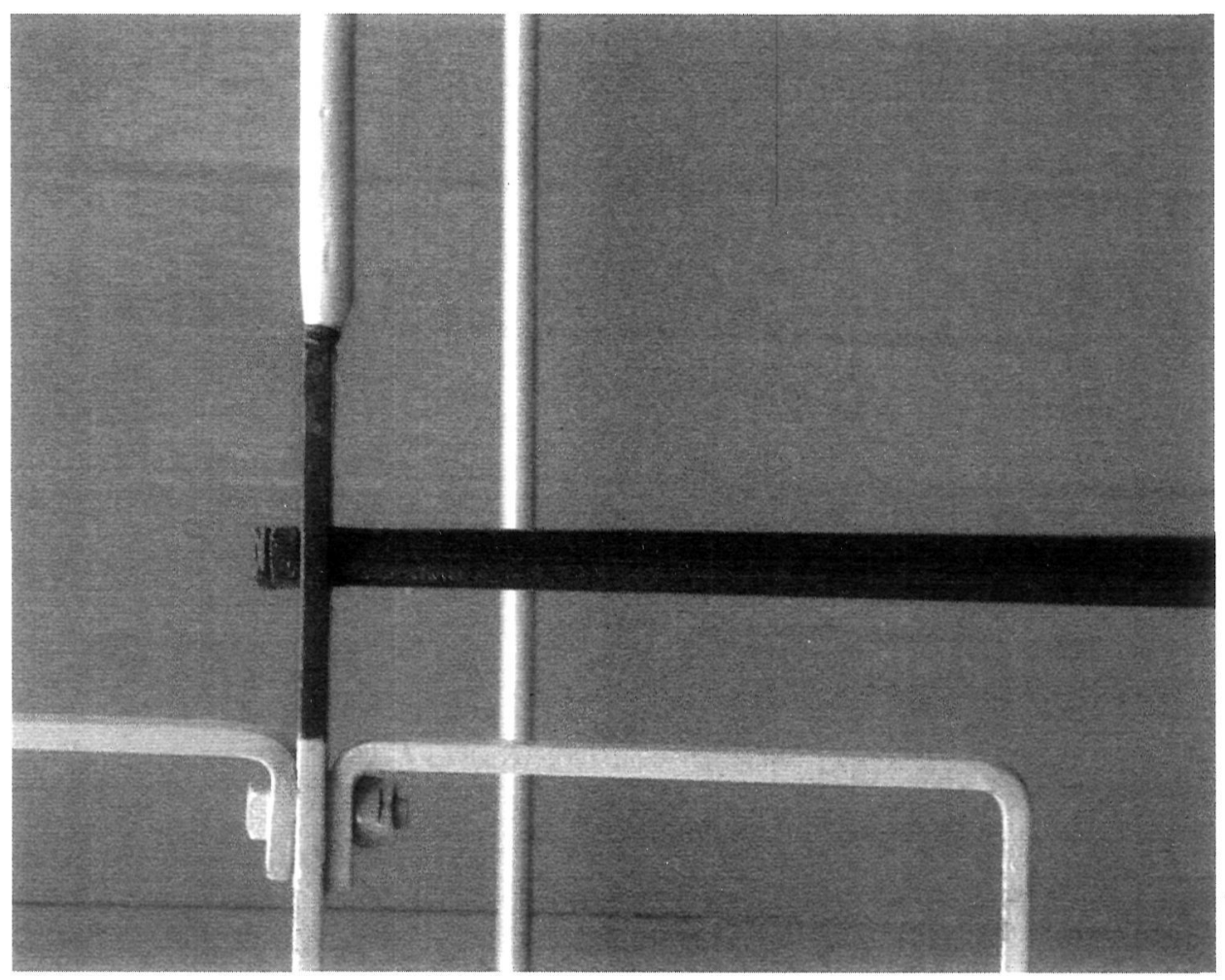

${ }^{20} \mathrm{~J}$. Aróstegui, presentó la ponencia La transición a la democracia en España: una historiografía mejorable en el congreso Historia de la Transición en España, el día 13 de enero de 2000 en Almeria, donde puso de relieve la necesidad de investigar en la etapa de la Transición sobre todo a nivel de provincias y municipios para poder "construir esqueletos" que nos permitan posteriores estudios comparativos. 